\title{
Does deinstitutionalization work? Relationships between psychiatric outpatient and inpatient care provision in a rural German catchment area
}

\author{
J uan Valdes-Stauber ${ }^{1}$, Michael von Cranach ${ }^{2}$, Albert Putzhammer ${ }^{2}$, Reinhold Kilian ${ }^{3}$ \\ 1. Zentrum für Psychiatrie Südwürttemberg, Department of Psychiatry I, University of Ulm, Germany. \\ 2. Bezirkskrankenhaus Kaufbeuren, Germany. 3. Bezirkskrankenhaus Günzburg, Section Epidemiology Research, \\ Department of Psychiatry II, University of UIm, Germany
}

Correspondence: J uan Valdes-Stauber. Address: Zentrum für Psychiatrie Südwürttemberg, Weingartshoferstr. 2, 88214 Ravensburg, Germany. E-mail: juan.valdes-stauber@zfp-zentrum.de

Received: December 19, 2012

DOI : $10.5430 /$ jha.v2n4p48
Accepted: May 2, 2013

URL: http://dx.doi.org/10.5430/jha.v2n4p48

\section{Abstract}

Objective: We intended to find out whether an intensification of outpatient care after conclusion of the deinstitutionalization era in the year 2001 had an influence on number of hospitalizations, readmission figures, length of inpatient -stay (LOS), cumulative length of inpatient-stay (cumulative LOS) and coercive measures.

Method: We investigated the development of 17 inpatient and outpatient variables for 2002-10 within a district psychiatric hospital responsible for a rural catchment area of 320,000 inhabitants. The sample consisted of 31,537 inpatient admissions and 35,372 outpatients accounted on an annual basis. Some figures were compared with those at state and federal levels. Associations between aggregated hospitalization and outpatient care variables were assessed by means of robust bivariate Prais-Winsten regression models for time series.

Results: Over the surveyed period, number of admissions, admitted individuals, and hospitalization rates remained stable, contrary to state and federal tendencies. Cumulative LOS and readmission figures decreased, whereas average LOS increased. Number of admissions, cumulative LOS, and readmission figures were negatively associated with number of outpatients treated which increased over the surveyed period. Number of coercive measures decreased, numbers of involuntary admissions under the guardianship law remained lower than at federal and state levels, and ambulatory activity remained three times higher than in the state in which the surveyed catchment area is located.

Conclusions: Community-oriented ambulatory care on the basis of multi-disciplinary assertive teams seems to be able to reduce readmissions and cumulative length of inpatient-stay, while keeping at the same time the number of admissions and coercive measures stable. Economic and clinical effects on real inpatient care, however, cannot be definitively evaluated as long as bed provision does not decrease proportionally with the increase of ambulatory activity.

\section{Key words}

Psychiatric outpatient clinic, Psychiatric hospitalizations, Readmissions in psychiatry, Length of inpatient-stay in psychiatry, Involuntary admissions, Coercive measures 


\section{I ntroduction}

Outpatient clinics have been little evaluated despite their importance as standard components of community mental health systems. Outpatient care projects, which started in the 1960s, have become more important in terms of the deinstitutionalization process of large asylums, resulting in a change from a somewhat custodial to a community-based treatment plan. As in other Western countries, the number of psychiatric beds in psychiatric hospitals as well as psychiatric departments in general hospitals decreased in Germany between 1970 and 1988 from 160 to 113 per 100,000 inhabitants (about 29\%) ${ }^{[1]}$; in the nineties bed rates remained stable ${ }^{[2,3]}$, whereas between 1999 and 2009 a slight increase can be noticed (81 vs. 92 beds per 100,000 inhabitants) ${ }^{[4]}$ owing to an increase in psychosomatic as well as rehabilitation beds. The mean length of inpatient-stay decreased between 1975 and 2003 by about 54\% ${ }^{[2]}$, and between 1998 and 2009 it decreased from 38.4 to 20.4 inpatient days (about 47\%), whereas the rate of psychiatric admissions increased in the same period from 94.6 to 140 per 1,000 inhabitants (about $48 \%)^{[5]}$.

The German National Health System is built on dichotomous principles: medical and social services, hospital and ambulatory care, rehabilitation and acute treatment are based on different statutory, funding and management structures. Ambulatory care in psychiatry is separate from psychotherapy and psychosomatic care; ambulatory psychiatric care is provided by private practitioners funded by the public, and are rarely networked with hospitals. Owing to the inadequacy of office-based psychiatric assistance in terms of meeting the needs of chronically mentally-ill people, the German federal government decided in 1975 to create multidisciplinary outpatient clinics networked with psychiatric hospitals and psychiatric departments situated in general hospitals as a necessary complement to single-handed private practice (see first representative survey of Federal Joint Commission of Stakeholders of Psychiatric Hospitals ${ }^{[6]}$ ). In Germany, up to $90 \%$ of outpatient care is provided by office-based psychiatrists, who rarely offer home-based or multidisciplinary intervention. Outpatient clinics (PIAs) have provided since the 1980s wide community-based care for people suffering from severe mental disorders, who often show non-adherence, by fulfilling the tasks of both outpatient clinics and community mental health teams ${ }^{[7]}$.

There is some evidence that outpatient service contracts are based on therapeutic relationships and continuity of care, but community-based teams may be better equipped to ensure attendance at outpatient appointments because of their innovative multidisciplinary interventions ${ }^{[8]}$. Some studies indicate that outpatient services provided by community mental health teams are more successful ${ }^{[9,10]}$ owing to their home-based and assertive care principles. German psychiatric care provision, however, does not differentiate between outpatient clinics and community mental health teams, differently from English-speaking or Scandinavian countries.

In this study, we aim to assess the possible influences of outpatient activity on inpatient length of stay and coercive outcomes. The data at the regional and national level would suggest that deinstitutionalization is usually associated with the reduction of acute ward beds, a reduction in mean length of inpatient-stay but with an increase of admissions, readmissions, involuntary admissions and long-term beds ${ }^{[11]}$. The key question is whether an intensification of ambulatory care could reduce hospitalizations and readmissions and keep mean length of inpatient-stay, cumulative length of stay, and number of coercive measures at a low level.

\section{Evaluation of ambulatory care settings in psychiatry}

Investigations of the relationships between ambulatory psychiatric care and readmission risk as well as length of stay in psychiatric wards show disparate results. Drawing boundaries between different ambulatory settings such as assertive community treatment, home-based treatment, day clinics, and care provision by outpatient clinics is difficult. Burns et al. ${ }^{[12]}$ conclude on the basis of a meta-analysis that the evidence for home treatment compared with other community-based services is not strong, although it reduces days spent in hospital compared with inpatient treatment alone. There is evidence that visiting patients at home regularly and taking responsibility for both health and social care can reduce days in hospital ${ }^{[12]}$. Marshall \& Lockwood ${ }^{[13]}$ demonstrate in another meta-analysis that patients receiving assertive community 
treatment (ACT) are more likely to remain in contact with services than people receiving standard community care and are significantly less likely to be admitted to hospital than those receiving hospital-based rehabilitation. Therefore, ACT invariably reduces the cost of hospital care, but does not have a clear financial advantage over standard care when other costs are taken into account. According to a review of Marshall et al. ${ }^{[14]}$, there is no evidence that day-treatment programmes are superior to continuing outpatient care in terms of improving psychiatric symptoms, social outcome, or costs. A French prospective and comparative cohort study over a five-year period demonstrated a significant immediate decrease in both number of admissions and duration of hospital stay after the provision of a mobile crisis intervention team ${ }^{[15]}$. From the second year onwards, however, the use of hospitalization did not seem to be influenced by the type of care initially given to the patient ${ }^{[15]}$. Furthermore, from a legal perspective, an Israeli study examined the risk of readmission and demonstrated that the probability of readmission of court-ordered and psychiatrist-ordered groups was significantly lower than that of voluntarily admitted patients, possibly related to a longer length of inpatient-stay ${ }^{[16]}$. Burgess et al. ${ }^{[17]}$ argued that the community treatment orders (CTOs) used in Australia on discharge from first admission to hospital were associated with lower readmission risk. For patients at risk of beginning a career of long-term psychiatric hospitalization, sole reliance on community-initiated orders appeared to prevent additional hospital involvement ${ }^{[18]}$. Tyrer et al. ${ }^{[19]}$ compared the clinical outcome and costs of care of psychiatric patients in London allocated to community-based multidisciplinary teams or to hospital-based outpatient care programmes after discharge from inpatient care; the clinical outcomes were similar but admission to hospital during follow-up was more likely in the hospital-based care group. In general, lack of 24-hour emergency settings correlates positively with the use of inpatient care alone ${ }^{[20]}$. It indicates that the extended availability of outpatient services in the community for people in crisis may facilitate the comprehensive utilization of mental health services ${ }^{[8]}$.

\section{Description of the catchment area}

The catchment area of the regional psychiatric hospital which we surveyed downsized between 1970 and 2000 from 940,000 to 320,000 inhabitants; hospital beds were reduced from 1,180 to 200 (including day-hospital places, excluding forensic beds) in the same period of time. This process was possible on the basis of consecutive decentralization steps taken between 1984 and 1999 as well as the installation of a community-based outpatient clinic. The bed index decreased, however, from 12.5 in 1970 to 5.9 per 10,000 inhabitants thirty-five years later, especially after conclusion of the deinstitutionalization process of long-stay patients (calculation conducted by the authors).

The catchment area in Southern Bavaria shows a rural structure and covers approximately 320,000 inhabitants. The regional hospital in charge consists of three clinics: one for general psychiatry, one for forensic psychiatry and one for neurology. The clinic for general psychiatry is divided into five departments: acute general psychiatry, psychotherapy, geriatrics, substance abuse disorders, comorbidity of mental retardation and general psychiatric disorders. In addition, the hospital has an outpatient clinic consisting of nine specialized teams, including two decentralized care teams. The number of annual hospitalizations amounts to 3,500, corresponding to 2,000 individuals. The hospital provides 5.5 acute beds per 10,000 inhabitants. The deinstitutionalization process was completed in 2001. The outpatient clinic takes care of more than 4,600 individuals per year, all of them suffering from severe mental disorders, and is in addition responsible for psychiatric emergencies. Cooperation with the guardianship courts and judges, local police, legal guardians, the general hospital in the neighborhood, sheltered facilities, ambulatory nurse teams and general practitioners is seen as very effective. Mood disorders are the most frequent diagnosis (approximately 30\%) followed by schizophrenia (25\%), dementia (18\%), substance abuse disorders (12\%), and borderline and complex post-traumatic stress disorders. The outpatient clinic staff comprises approximately 50 professionals from seven different professions working in multidisciplinary teams. There are at least two professionals responsible for each patient ('therapeutic case managers') to ensure continuity of relationships. Five clinical conferences are held every week, one of them in cooperation with the acute ward, and continuous education every two weeks improves care quality. The outpatient clinic is embedded in a coordinated regional community network and participates in the decision-making concerning the discharge process. 
Several accreditations by specialized agencies have confirmed the excellence of the clinical and organizational tasks performed. Protocols, quarterly and annual reviews and an ambitious supporting intranet portal help to provide balanced support in cooperation with the authorities and the social services in the community.

Table 1. Development of inpatient and outpatient care variables (2002-10).

\begin{tabular}{|c|c|c|c|c|c|c|c|c|c|}
\hline Census year & 2002 & 2003 & 2004 & 2005 & 2006 & 2007 & 2008 & 2009 & 2010 \\
\hline $\begin{array}{l}\text { Number of annual } \\
\text { admissions }\end{array}$ & 3,582 & 3,381 & 3,482 & 3,464 & 3,468 & 3,421 & 3,589 & 3,559 & 3,411 \\
\hline $\begin{array}{l}\text { Number of annually } \\
\text { admitted individuals }\end{array}$ & 2,031 & 1,960 & 1,930 & 2,038 & 2,045 & 2,125 & 2,233 & 2,156 & 2,037 \\
\hline $\begin{array}{l}\text { Admission ratio } \\
\text { (admissions/individuals) }\end{array}$ & 1.76 & 1.72 & 1.80 & 1.70 & 1.69 & 1.61 & 1.61 & 1.65 & 1.67 \\
\hline LOS per admission (days) & 19.22 & 19.59 & 20.18 & 20.14 & 20.35 & 21.48 & 21.9 & 21.21 & 21.54 \\
\hline $\mathrm{M}(\mathrm{SD})$ & $(28.62)$ & $(27.31)$ & (29.19) & $(27.25)$ & $(26.71)$ & $(28.25)$ & (29.18) & $(27.79)$ & $(27.13)$ \\
\hline $\begin{array}{l}\text { Average annual cumulative } \\
\text { inpatient-LOS per } \\
\text { individual (days) }\end{array}$ & 47.74 & 43.57 & 47.48 & 41.37 & 43.93 & 41.46 & 40.63 & 45.35 & 39.98 \\
\hline Age M (SD) & $\begin{array}{l}42.9 \\
(18.1)\end{array}$ & $\begin{array}{l}43.1 \\
(18.1)\end{array}$ & $\begin{array}{l}43.8 \\
(17.6)\end{array}$ & $\begin{array}{l}44.4 \\
(17.9)\end{array}$ & $\begin{array}{l}45.5 \\
(18.6)\end{array}$ & $\begin{array}{l}45.7 \\
(17.9)\end{array}$ & $\begin{array}{l}45.7 \\
(18.1)\end{array}$ & $\begin{array}{l}46.6 \\
(18.1)\end{array}$ & $\begin{array}{l}45.8 \\
(17.6)\end{array}$ \\
\hline Proportion of women (\%) & $57.8 \%$ & $57.6 \%$ & $58.1 \%$ & $58.0 \%$ & $56.9 \%$ & $55.5 \%$ & $56.9 \%$ & $55.9 \%$ & $55.1 \%$ \\
\hline $\begin{array}{l}\text { Proportion of admitted } \\
\text { individuals in charge of } \\
\text { outpatient clinic (\%) }\end{array}$ & - & - & - & $48.9 \%$ & $53.7 \%$ & $51.1 \%$ & $49.1 \%$ & $49.2 \%$ & $52.1 \%$ \\
\hline $\begin{array}{l}\text { Treatment periods at } \\
\text { outpatient clinic for whole } \\
\text { sample M (SD) }\end{array}$ & - & - & - & $\begin{array}{l}1.11 \\
(1.28)\end{array}$ & $\begin{array}{l}1.45 \\
(1.63)\end{array}$ & $\begin{array}{l}1.40 \\
(1.64)\end{array}$ & $\begin{array}{l}1.30 \\
(1.58)\end{array}$ & $\begin{array}{l}1.29 \\
(1.57)\end{array}$ & $\begin{array}{l}1.43 \\
(1.65)\end{array}$ \\
\hline $\begin{array}{l}\text { Annual number of } \\
\text { outpatients treated }\end{array}$ & 3,736 & 3,866 & 3,648 & 3,838 & 3,712 & 4,035 & 3,924 & 3,957 & 4,656 \\
\hline $\begin{array}{l}\text { Proportion of readmitted } \\
\text { individuals (\%) }\end{array}$ & $43 \%$ & $42 \%$ & $44 \%$ & $41 \%$ & $41 \%$ & $38 \%$ & $38 \%$ & $39 \%$ & $37 \%$ \\
\hline $\begin{array}{l}\text { Average number of } \\
\text { readmissions per readmitted } \\
\text { individual }\end{array}$ & 2.48 & 2.22 & 2.35 & 2.05 & 2.15 & 1.93 & 1.85 & 2.14 & 1.85 \\
\hline $\begin{array}{l}\text { Proportion of frequently } \\
\text { readmitted individuals } \\
(>=3)\end{array}$ & $26 \%$ & $24 \%$ & $26 \%$ & $22 \%$ & $23 \%$ & $20 \%$ & $18 \%$ & $20 \%$ & $18 \%$ \\
\hline $\begin{array}{l}\text { Proportion of involuntary } \\
\text { admissions under the } \\
\text { guardianship law (\%) }\end{array}$ & $1.83 \%$ & $0.97 \%$ & $1.25 \%$ & $0.91 \%$ & $0.58 \%$ & $0.81 \%$ & $2.58 \%$ & $3.0 \%$ & $3.0 \%$ \\
\hline $\begin{array}{l}\text { Number of coercive } \\
\text { measures per hospitalization }\end{array}$ & 0.25 & 0.26 & 0.29 & 0.25 & 0.34 & 0.24 & 0.16 & 0.16 & 0.19 \\
\hline
\end{tabular}

Percentages for categorical variables; mean (M) and standard deviation (SD) for continuous variables.

\subsection{Objectives}

The main objective of this study was to investigate possible effects of intensity of outpatient care on inpatient treatment variables in a rural catchment area covering 320,000 inhabitants a decade after completed deinstitutionalization of long-stay patients in the year 2001.

We first describe the development of administrative, clinical, and inpatient as well as outpatient care variables for the period after conclusion of the deinstitutionalization process (2002-10) on the basis of individual data. 


\begin{tabular}{|c|c|c|c|c|c|c|c|c|c|c|c|c|}
\hline $\begin{array}{l}\text { ICD-10/ } \\
\text { Census year }\end{array}$ & & F0 & F1 & F2 & F3 & F4 & F5 & F6 & F7 & F8 & F9 & $\begin{array}{l}\text { Total } \\
\text { (annual) }\end{array}$ \\
\hline 2002 & $\begin{array}{l}\text { S.A. } \\
\text { GER }\end{array}$ & $\begin{array}{l}114(3.2 \%) \\
84,731 \\
(8.6 \%)\end{array}$ & $\begin{array}{l}1,787(50 \%) \\
366,957 \\
(37.2 \%)\end{array}$ & $\begin{array}{l}537(15 \%) \\
137,027 \\
(13.9 \%)\end{array}$ & $\begin{array}{l}663(18.5 \%) \\
161,320 \\
(16.4 \%)\end{array}$ & $\begin{array}{l}266(7.4 \%) \\
137,213 \\
(13.9 \%)\end{array}$ & $\begin{array}{l}5(0.14 \%) \\
19,513 \\
(2.0 \%)\end{array}$ & $\begin{array}{l}181(5 \%) \\
37,978 \\
(3.8 \%)\end{array}$ & $\begin{array}{l}10(0.3) \\
6,837 \\
(0.7 \%)\end{array}$ & $\begin{array}{l}8(0.2) \\
6,857 \\
(0.7 \%)\end{array}$ & $\begin{array}{l}5(0.14 \%) \\
23,627 \\
(2.4 \%)\end{array}$ & $\begin{array}{l}3,576 \\
986,573 \\
(100 \%)\end{array}$ \\
\hline 2003 & $\begin{array}{l}\text { S.A. } \\
\text { GER }\end{array}$ & $\begin{array}{l}118(3.5 \%) \\
82,748 \\
(8.3 \%)\end{array}$ & $\begin{array}{l}1,719(51 \%) \\
368,774 \\
(37.1 \%)\end{array}$ & $\begin{array}{l}541(16 \%) \\
137,882 \\
(13.9 \%)\end{array}$ & $\begin{array}{l}644(19 \%) \\
169,405 \\
(17.1 \%)\end{array}$ & $\begin{array}{l}210(6.2 \%) \\
137,452 \\
(13.8 \%)\end{array}$ & $\begin{array}{l}2(0.06 \%) \\
17,987 \\
(1.8 \%)\end{array}$ & $\begin{array}{l}132(3.9 \%) \\
38,084 \\
(3.8 \%)\end{array}$ & $\begin{array}{l}6(0.2 \%) \\
6,874 \\
(0.7 \%)\end{array}$ & $\begin{array}{l}5(0.15 \%) \\
6,289 \\
(0.6 \%)\end{array}$ & $\begin{array}{l}3(0.09 \%) \\
23,920 \\
(2.4 \%)\end{array}$ & $\begin{array}{l}3,380 \\
993,732 \\
(100 \%)\end{array}$ \\
\hline 2004 & $\begin{array}{l}\text { S.A. } \\
\text { GER }\end{array}$ & $\begin{array}{l}116(4.8 \%) \\
85,096 \\
(8.4 \%)\end{array}$ & $\begin{array}{l}1,700(48.8 \%) \\
373,529 \\
(36.6 \%)\end{array}$ & $\begin{array}{l}550(15.8 \%) \\
138,129 \\
(13.5 \%)\end{array}$ & $\begin{array}{l}680(19.5 \%) \\
181,141 \\
(17.8 \%)\end{array}$ & $\begin{array}{l}187(5.4 \%) \\
142,123 \\
(13.9 \%)\end{array}$ & $\begin{array}{l}14(0.4 \%) \\
17,020 \\
(1.7 \%)\end{array}$ & $\begin{array}{l}182(5.2 \%) \\
38,759 \\
(3.8 \%)\end{array}$ & $\begin{array}{l}0 \\
6,865 \\
(0.7 \%)\end{array}$ & $\begin{array}{l}1(0.03 \%) \\
5,675 \\
(0.6 \%)\end{array}$ & $\begin{array}{l}1(0.03 \%) \\
26,193 \\
(2.6 \%)\end{array}$ & $\begin{array}{l}3,481 \\
1,019,154 \\
(100 \%)\end{array}$ \\
\hline 2005 & $\begin{array}{l}\text { S.A. } \\
\text { GER }\end{array}$ & $\begin{array}{l}111(3.2 \%) \\
88,671 \\
(8.5 \%)\end{array}$ & $\begin{array}{l}1,610(46.5 \%) \\
386,470 \\
(36.9 \%)\end{array}$ & $\begin{array}{l}525(15.2 \%) \\
138,470 \\
(13.2 \%)\end{array}$ & $\begin{array}{l}817(23.6 \%) \\
191,451 \\
(18.3 \%)\end{array}$ & $\begin{array}{l}168(4.8 \%) \\
142,931 \\
(13.7 \%)\end{array}$ & $\begin{array}{l}13(0.4 \%) \\
16,620 \\
(1.6 \%)\end{array}$ & $\begin{array}{l}210(6.1 \%) \\
39,083 \\
(3.7 \%)\end{array}$ & $\begin{array}{l}2(0.06 \%) \\
6,855 \\
(0.6 \%)\end{array}$ & $\begin{array}{l}4(0.12 \%) \\
5,757 \\
(0.5 \%)\end{array}$ & $\begin{array}{l}2(0.06 \%) \\
26,081 \\
(2.5 \%)\end{array}$ & $\begin{array}{l}3,462 \\
1,046,365 \\
(100 \%)\end{array}$ \\
\hline 2006 & $\begin{array}{l}\text { S.A. } \\
\text { GER }\end{array}$ & $\begin{array}{l}216(3.2 \%) \\
89,867 \\
(8.5 \%)\end{array}$ & $\begin{array}{l}1,533(44.2 \%) \\
385,147 \\
(36.4 \%)\end{array}$ & $\begin{array}{l}477(13.7 \%) \\
137,171 \\
(13.0 \%)\end{array}$ & $\begin{array}{l}854(24.6 \%) \\
200,791 \\
(19.0 \%)\end{array}$ & $\begin{array}{l}151(4.4 \%) \\
142,704 \\
(13.5 \%)\end{array}$ & $\begin{array}{l}5(0.14 \%) \\
16,995 \\
(1.6 \%)\end{array}$ & $\begin{array}{l}228(6.6 \%) \\
40,397 \\
(3.8 \%)\end{array}$ & $\begin{array}{l}2(0.06) \\
6,795 \\
(0.6 \%)\end{array}$ & $\begin{array}{l}0 \\
6,072 \\
(0.6 \%)\end{array}$ & $\begin{array}{l}1(0.03 \%) \\
27,377 \\
(2.6 \%)\end{array}$ & $\begin{array}{l}3,467 \\
1,057,564 \\
(100 \%)\end{array}$ \\
\hline 2007 & $\begin{array}{l}\text { S.A. } \\
\text { GER }\end{array}$ & $\begin{array}{l}180(5.3 \%) \\
92,012 \\
(8.4 \%)\end{array}$ & $\begin{array}{l}1,338(39.1 \%) \\
404,290 \\
(37.0 \%)\end{array}$ & $\begin{array}{l}489(14.3 \%) \\
136,523 \\
(12.5 \%)\end{array}$ & $\begin{array}{l}944(27.6 \%) \\
213,026 \\
(19.5 \%)\end{array}$ & $\begin{array}{l}214(6.3 \%) \\
144,656 \\
(13.2 \%)\end{array}$ & $\begin{array}{l}15(0.4 \%) \\
16,565 \\
(1.5 \%)\end{array}$ & $\begin{array}{l}227(6.6 \%) \\
40,598 \\
(3.7 \%)\end{array}$ & $\begin{array}{l}7(0.2 \%) \\
7,001 \\
(0.6 \%)\end{array}$ & $\begin{array}{l}2(0.06 \%) \\
5,939 \\
(0.5 \%)\end{array}$ & $\begin{array}{l}2(0.06 \%) \\
28,958 \\
(2.7 \%)\end{array}$ & $\begin{array}{l}3,418 \\
1,093,641 \\
(100 \%)\end{array}$ \\
\hline 2008 & $\begin{array}{l}\text { S.A. } \\
\text { GER }\end{array}$ & $\begin{array}{l}201(5.6 \%) \\
93,870 \\
(8.3 \%)\end{array}$ & $\begin{array}{l}1,399(39 \%) \\
424,660 \\
(37.6 \%)\end{array}$ & $\begin{array}{l}491(13.7 \%) \\
136,246 \\
(12.1 \%)\end{array}$ & $\begin{array}{l}999(27.8 \%) \\
222,386 \\
(19.7 \%)\end{array}$ & $\begin{array}{l}246(6.8 \%) \\
145,562 \\
(12.9 \%)\end{array}$ & $\begin{array}{l}10(0.3 \%) \\
16,400 \\
(1.5 \%)\end{array}$ & $\begin{array}{l}235(6.5 \%) \\
40,872 \\
(3.6 \%)\end{array}$ & $\begin{array}{l}2(0.06 \%) \\
7,247 \\
(0.6 \%)\end{array}$ & $\begin{array}{l}0 \\
6,713 \\
(0.6 \%)\end{array}$ & $\begin{array}{l}4(0.11 \%) \\
29,703 \\
(1.6 \%)\end{array}$ & $\begin{array}{l}3,587 \\
1,127,971 \\
(100 \%)\end{array}$ \\
\hline 2009 & $\begin{array}{l}\text { S.A. } \\
\text { GER }\end{array}$ & $\begin{array}{l}315(8.9 \%) \\
94,239 \\
(8.2 \%)\end{array}$ & $\begin{array}{l}1,362(38.5 \%) \\
431,163 \\
(37.4 \%)\end{array}$ & $\begin{array}{l}488(13.8 \%) \\
135,713 \\
(11.3 \%)\end{array}$ & $\begin{array}{l}886(25.6 \%) \\
237,242 \\
(20.6 \%)\end{array}$ & $\begin{array}{l}235(6.6 \%) \\
147,900 \\
(12.8 \%)\end{array}$ & $\begin{array}{l}0 \\
15,976 \\
(1.4 \%)\end{array}$ & $\begin{array}{l}243(6.9 \%) \\
40,665 \\
(3.5 \%)\end{array}$ & $\begin{array}{l}4(0.1 \%) \\
7,323 \\
(0.6 \%)\end{array}$ & $\begin{array}{l}0 \\
6,726 \\
(0.6 \%)\end{array}$ & $\begin{array}{l}2(0.06 \%) \\
30,318 \\
(2.6 \%)\end{array}$ & $\begin{array}{l}3,535 \\
1,151,390 \\
(100 \%)\end{array}$ \\
\hline 2010 & $\begin{array}{l}\text { S.A. } \\
\text { GER }\end{array}$ & $\begin{array}{l}276(8.1 \%) \\
94,950 \\
(8.2 \%)\end{array}$ & $\begin{array}{l}1,470(43.4 \%) \\
427,308 \\
(36.7 \%)\end{array}$ & $\begin{array}{l}392(11.6 \%) \\
131,469 \\
(11.3 \%)\end{array}$ & $\begin{array}{l}793(23.4 \%) \\
254,455 \\
(21.9 \%)\end{array}$ & $\begin{array}{l}241(7.1 \%) \\
150,207 \\
(12.9 \%)\end{array}$ & $\begin{array}{l}22(0.6 \%) \\
16,044 \\
(1.4 \%)\end{array}$ & $\begin{array}{l}151(4,5 \%) \\
40,193 \\
(3.5 \%)\end{array}$ & $\begin{array}{l}20(0.6 \%) \\
7,059 \\
(0.6 \%)\end{array}$ & $\begin{array}{l}10(0.3 \%) \\
6,736 \\
(0.6 \%)\end{array}$ & $\begin{array}{l}14(0.4 \%) \\
30,632 \\
(2.6 \%)\end{array}$ & $\begin{array}{l}3,389 \\
1,163,613 \\
(100 \%)\end{array}$ \\
\hline $\begin{array}{l}\text { Total } \\
\text { (Survey } \\
\text { Period) }\end{array}$ & S.A. & $\begin{array}{l}1,687 \\
(5.4 \%) \\
806,184 \\
(8.4 \%)\end{array}$ & $\begin{array}{l}13,918 \\
(44.5 \%) \\
3,568,298 \\
(37 \%)\end{array}$ & $\begin{array}{l}4,490 \\
(14.4 \%) \\
1,228,657 \\
(12.7 \%)\end{array}$ & $\begin{array}{l}7,280 \\
(23.3 \%) \\
1,831,217 \\
(20 \%)\end{array}$ & $\begin{array}{l}1,918 \\
(6.1 \%) \\
1,148,625 \\
(11.9 \%)\end{array}$ & $\begin{array}{l}86 \\
(0.3 \%) \\
153,080 \\
(1.6 \%)\end{array}$ & $\begin{array}{l}1,789 \\
(5.7 \%) \\
322,353 \\
(3.3 \%)\end{array}$ & $\begin{array}{l}53 \\
(0.2 \%) \\
62,856 \\
(0.6 \%)\end{array}$ & $\begin{array}{l}30 \\
(0.1 \%) \\
56,764 \\
(0.6 \%)\end{array}$ & $\begin{array}{l}34 \\
(0.1 \%) \\
246,809 \\
(2.6 \%)\end{array}$ & $\begin{array}{l}31,295 \\
(100 \%) \\
9,640,003 \\
(100 \%)\end{array}$ \\
\hline
\end{tabular}

Absolute figures (percentage). S.A.= Surveyed area; GER= Federal level (Germany)

In a second step, we investigated the association between the number of annually treated outpatients and the mean length of inpatient-stay, considering total number of annual admissions, for the period after the installation of the assessed outpatient clinic (1981-2010).

In a further step, we investigated the development of the annual number of outpatients treated as well as the development of six inpatient variables (number of admissions, average length of inpatient-stay, average annual cumulative length of inpatient-stay per individual, proportion of readmitted individuals, average number of readmissions per readmitted patient, and proportion of frequently readmitted individuals) in the sample as well as for each principal diagnostic group. Finally, we investigated possible associations between the number of outpatients treated and the six mentioned hospitalization variables.

The status of certain variables (admission rates, average length of inpatient-stay, proportion of involuntary admissions under the guardianship law, and rates of treatment periods by outpatient clinic) can be compared with general tendencies in the state in which the catchment area is situated (Bavaria) as well as in Germany by means of figures calculated by the authors.

\subsection{Method}

This study is based on aggregated data of 31,537 inpatient admissions and 35, 372 outpatients accounted on an annual basis, covering a period of nine years from 2002 to 2010. Data were aggregated on an annual basis by primary ICD-10 diagnostic groups.

We considered 17 variables grouped as follows (see Tables 1 to 3): 
(1) Administrative variables: number of annual admissions, number of individuals admitted annually, admission ratio, average length of inpatient-stay, and average annual cumulative length of inpatient-stay per individual.

(2) Socio-demographic variables: age and gender.

(3) Clinical variables: principal diagnostic groups according to the International Classification of Mental Disorders (ICD-10).

(4) Outpatient care variables: annual number of outpatients treated, proportion of admitted individuals in the charge of the surveyed outpatient clinic, annual treatment periods by outpatient clinic, and prevalence of treatment periods by outpatient clinic per $10^{3}$ inhabitants and year.

(5) Inpatient variables: proportion of readmitted individuals, average number of annual readmissions per readmitted individual, and proportion of hospitalized individuals with frequent readmissions (when $>=3$ readmissions).

(6) Legal variables: proportion or involuntary admissions under the guardianship law, and number of coercive measures per hospitalization.

Table 3. Comparison of administrative and care variables at regional, state and federal level.

\begin{tabular}{|c|c|c|c|c|c|c|c|c|c|c|}
\hline \multicolumn{2}{|l|}{ Census year } & 2002 & 2003 & 2004 & 2005 & 2006 & 2007 & 2008 & 2009 & 2010 \\
\hline \multicolumn{11}{|c|}{ Annual psychiatric admission rates per $10^{3}$ inhabitants } \\
\hline \multicolumn{2}{|l|}{ Germany } & 11.9 & 12.0 & 12.3 & 12.7 & 12.8 & 13.3 & 13.7 & 14.1 & 14.2 \\
\hline \multicolumn{2}{|l|}{ Bavaria (BY) } & 11.6 & 11.5 & 11.9 & 12.1 & 12.3 & 13.0 & 13.5 & 13.4 & 14.4 \\
\hline \multicolumn{2}{|c|}{ Surveyed catchment area in } & 11.2 & 10.5 & 10.8 & 10.7 & 10.7 & 10.6 & 11.2 & 11.1 & 10.6 \\
\hline \multicolumn{11}{|l|}{ Ave } \\
\hline \multicolumn{2}{|l|}{ Germany } & 22.7 & 22.8 & 21.4 & 20.8 & 20.9 & 20.8 & 20.3 & 20.3 & 20.3 \\
\hline \multicolumn{2}{|l|}{ Bavaria (BY) } & 22.9 & 23.1 & 22.0 & 21.6 & 22.2 & 22.0 & 21.7 & 21.5 & 21.4 \\
\hline \multicolumn{2}{|c|}{$\begin{array}{l}\text { Surveyed catchment area in } \\
\text { BY }\end{array}$} & 19.2 & 19.6 & 20.2 & 20.1 & 20.3 & 21.5 & 21.9 & 21.2 & 21.5 \\
\hline \multicolumn{11}{|c|}{ Average length of inpatient-stay according to principal diagnostic groups (days) } \\
\hline \multirow{2}{*}{$\begin{array}{l}\text { Organic mental } \\
\text { disorders (F0) }\end{array}$} & S.A. & 18.9 & 18.5 & 21.5 & 23.8 & 19.5 & 26.3 & 18.6 & 18.1 & 21.1 \\
\hline & GER & 21.0 & 21.3 & 19.2 & 18.5 & 18.5 & 18.4 & 18.0 & 18.0 & 17.6 \\
\hline \multirow{2}{*}{$\begin{array}{l}\text { Addictive disorders } \\
\text { (F1) }\end{array}$} & S.A. & 11.5 & 11.8 & 10.9 & 11.1 & 11.1 & 11.3 & 10.9 & 11.0 & 12.1 \\
\hline & GER & 11.2 & 11.0 & 10.1 & 9.6 & 9.5 & 9.4 & 9.0 & 8.8 & 8.6 \\
\hline \multirow{2}{*}{ Schizophrenia (F2) } & S.A. & 31.9 & 30.5 & 29.4 & 29.4 & 28.1 & 28.5 & 28.4 & 25.5 & 28.9 \\
\hline & GER & 38.5 & 39.0 & 36.4 & 35.2 & 34.8 & 34.9 & 34.0 & 33.7 & 33.1 \\
\hline \multirow{2}{*}{$\begin{array}{l}\text { Mood disorders } \\
\text { (F3) }\end{array}$} & S.A. & 28.4 & 28.8 & 31.9 & 28.4 & 28.9 & 28.1 & 36.1 & 37.9 & 34.2 \\
\hline & GER & 35.4 & 35.4 & 33.8 & 33.5 & 33.6 & 33.6 & 33.6 & 33.7 & 33.6 \\
\hline \multirow{2}{*}{$\begin{array}{l}\text { Adjustment and } \\
\text { stress disorders (F4) }\end{array}$} & S.A. & 13.9 & 16.9 & 17.6 & 17.9 & 18.0 & 15.6 & 16.9 & 16.7 & 16.6 \\
\hline & GER & 19.2 & 19.2 & 17.8 & 17.4 & 17.3 & 17.3 & 17.2 & 17.1 & 17.1 \\
\hline \multirow{2}{*}{$\begin{array}{l}\text { Personality } \\
\text { disorders (F6) }\end{array}$} & S.A. & 17.0 & 20.2 & 23.4 & 25.4 & 22.9 & 22.1 & 27.3 & 22.6 & 23.7 \\
\hline & GER & 29.8 & 29.7 & 27.3 & 26.3 & 25.5 & 25.7 & 25.4 & 24.8 & 24.2 \\
\hline \multicolumn{11}{|c|}{ Proportion of involuntary admissions under the guardianship law (\% admissions) } \\
\hline \multicolumn{2}{|l|}{ Germany } & 4.37 & 4.55 & 4.38 & 4.40 & 4.47 & 4.68 & 4.72 & 4.75 & 4.79 \\
\hline \multicolumn{2}{|l|}{ Bavaria (BY) } & 9.4 & 10,10 & 9.75 & 9.60 & 8.90 & 9.30 & 9.70 & 10.17 & 9.34 \\
\hline \multicolumn{2}{|c|}{$\begin{array}{l}\text { Surveyed catchment area in } \\
\text { BY }\end{array}$} & 1.83 & 0.97 & 1.25 & 0.91 & 0.58 & 0.81 & 2.58 & 3.0 & 3.0 \\
\hline \multicolumn{11}{|c|}{ Prevalence of annual treatment periods by outpatient clinic per $10^{3}$ inhabitants and year } \\
\hline \multicolumn{2}{|l|}{ Bavaria (BY) } & 8.2 & 9.2 & 10.4 & 11.3 & 12.2 & 13.6 & 14.7 & 15.6 & 16.7 \\
\hline \multicolumn{2}{|c|}{$\begin{array}{l}\text { Surveyed catchment area in } \\
\text { BY }\end{array}$} & 29.2 & 31.7 & 30.4 & 30.4 & 31.8 & 32.1 & 35.1 & 38.2 & 42.5 \\
\hline
\end{tabular}

S.A.= Surveyed area; GER= Federal level (Germany); BY=Federal State of Bavaria. 
Individual data up to 2002 about the surveyed hospital were obtained from the IT Department and aggregated data up to 1981 from annual reviews by the outpatient clinic. Routine data for all admissions were grouped by census year, principal diagnosis and individuals. After statistical collapsing, data were treated anonymously.

Tendencies within the surveyed period of time and associations between inpatient and outpatient care variables were investigated for the whole sample as well as for diagnostic groups by means of bivariate Prais-Winsten regression analyses for time series. In regression models, robust regression coefficients were assessed in order to calculate confidence intervals.

Prais-Winsten regression ${ }^{[21,22]}$ was applied to control for autocorrelation in time series data. Autocorrelation was detected by means of the Durbin-Watson d coefficient ${ }^{[23]}$. The $d$ coefficient has a value range from zero to four, wherein an autocorrelation is indicated by the deviance of $\mathrm{d}$ from the value 2. Prais-Winsten regression models were estimated by the Prais module with STATA $12^{[24]}$. Random effects were not calculated, because data did not show a panel structure.

\section{Results}

A descriptive assessment of time series indicated that the number of annual admissions as well as annual number admitted individuals remained stable over the surveyed period, but admission ratio decreased about 0.1 , and share of readmitted individuals went from $43 \%$ down to $37 \%$, a decrease of about $26 \%$. Even average number of readmissions of readmitted individuals decreased from 2.48 to 1.85 , a drop of about $25 \%$. Proportion of frequently admitted individuals decreased from $26 \%$ to $18 \%$, a drop of about $31 \%$. On the other hand, average length of inpatient-stay increased about 2.3 days; conversely, average annual cumulative length of inpatient-stay per individual decreased from 47.74 to 39.98, a drop of about $16 \%$, within the same period. The sample became on average three years older, and the proportion of women decreased by $3.7 \%$. With regard to outpatient care, the number of annually treated outpatients increased over the survey period as did proportion of admitted individuals in the care of the outpatient clinic and average annual treatment periods per individual.

Figure 1. Relationship between admissions, LOS and outpatient activity at District Hospital of Kaufbeuren (1981-2010)

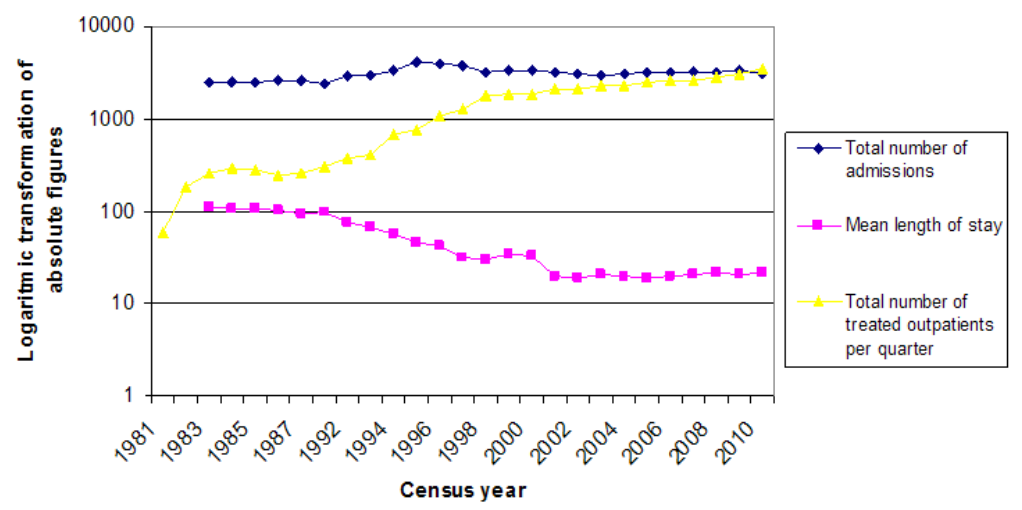

The time series for diagnostic distribution showed that addiction (F1) was the most frequent admission diagnosis, followed by mood disorders (F3), schizophrenia (F2), adjustment and stress disorders (F4), and organic mental disorders (F0). Other diagnostic groups were underrepresented. Some developments are remarkable: adjustment and stress disorders remained stable, mood disorders increased about $26 \%$, organic mental disorders almost tripled, and schizophrenia as well as addictions decreased (see Table 2). Diagnostic distribution showed significant differences over time (Chi-square test for diagnostic distribution $(\mathrm{df}=72)=825.55 ; p=0.000)$. Average length of inpatient-stay according to diagnosis remained quite stable for organic mental disorders, addictive disorders and adjustment and stress disorders, increased for affective and personality disorders and decreased for patients suffering from schizophrenia (see Table 3). 
The investigated association by means of a Prais-Winsten regression model between the average length of inpatient-stay and the annual number of patients in the charge of the outpatient clinic since its launch in 1981 showed a significant negative association between average length of inpatient-stay and number of outpatients treated $(b=-0.022 ; p=0.00012)$, even when the number of annual admissions was taken into account as control variable $(b=-0.018 ; p=0.00056)$ (Figure 1$)$.

Table 4. Prais-Winsten regression models for time series (relevant inpatient and outpatient care variables) on the basis of robust regression coefficients for the whole sample and according to principal diagnosis groups with time as regressor.

\begin{tabular}{|c|c|c|c|c|c|c|c|c|c|c|c|c|c|c|}
\hline \multirow{2}{*}{ Diagnosis } & \multicolumn{2}{|c|}{ Sample } & \multicolumn{2}{|l|}{ F0 } & \multicolumn{2}{|l|}{ F1 } & \multicolumn{2}{|l|}{ F2 } & \multicolumn{2}{|l|}{ F3 } & \multicolumn{2}{|l|}{ F4 } & \multicolumn{2}{|l|}{ F6 } \\
\hline & $\mathbf{b}$ & $\mathbf{p}$ & b & $\mathbf{p}$ & b & $\mathbf{p}$ & $\mathbf{p}$ & b & $\mathbf{b}$ & $\mathbf{p}$ & b & $\mathbf{p}$ & b & $\mathbf{p}$ \\
\hline $\begin{array}{l}\text { Dependent } \\
\text { variable }\end{array}$ & \multicolumn{14}{|c|}{ Annual number of outpatients treated } \\
\hline $\begin{array}{l}\text { Regressor: } \\
\text { Time }\end{array}$ & 67.31 & 0.038 & 46.06 & 0.000 & -32.8 & 0.001 & 13.2 & 0.094 & 58.4 & 0.037 & 11.6 & 0.125 & 12.57 & 0.001 \\
\hline Constant & 3,637 & 0.000 & 499 & 0.000 & 661 & 0.000 & 789 & 0.000 & 863 & 0.000 & 255 & 0.000 & 145 & 0.000 \\
\hline $\begin{array}{l}\text { Dependent } \\
\text { variable }\end{array}$ & \multicolumn{14}{|c|}{ Number of annual admissions } \\
\hline $\begin{array}{l}\text { Regressor: } \\
\text { Time }\end{array}$ & 5.23 & 0.544 & 28.8 & 0.000 & -39.1 & 0.045 & -15.6 & 0.000 & 37.7 & 0.045 & -8.2 & 0.391 & 7.7 & 0.243 \\
\hline Constant & 3,462 & 0.000 & 65.4 & 0.013 & 1,592 & 0.000 & 591 & 0.000 & 606 & 0.000 & 270 & 0.003 & 168 & 0.000 \\
\hline $\begin{array}{l}\text { Dependent } \\
\text { variable }\end{array}$ & \multicolumn{14}{|c|}{ Average number of readmissions } \\
\hline $\begin{array}{l}\text { Regressor: } \\
\text { Time }\end{array}$ & -0.06 & 0.001 & $-5 e-4$ & 0.929 & -0.76 & 0.009 & -0.20 & 0.410 & -0.036 & 0.001 & -0.04 & 0.021 & -0.09 & 0.145 \\
\hline Constant & 2.35 & 0.000 & 1.33 & 0.000 & 2.81 & 0.000 & 2.09 & 0.000 & 1.86 & 0.000 & 1.69 & 0.000 & 3.27 & 0.000 \\
\hline $\begin{array}{l}\text { Dependent } \\
\text { variable }\end{array}$ & \multicolumn{14}{|c|}{ Average length of inpatient-stay per hospitalization } \\
\hline $\begin{array}{l}\text { Regressor: } \\
\text { Time }\end{array}$ & 0.31 & 0.000 & 0.04 & 0.877 & -0.01 & 0.855 & -0.55 & 0.000 & 0.95 & 0.017 & 0.13 & 0.568 & 0.64 & 0.094 \\
\hline Constant & 19.4 & 0.000 & 20.6 & 0.000 & 11.3 & 0.000 & 31 & 0.000 & 27.6 & 0.000 & 16.1 & 0.000 & 20.2 & 0.000 \\
\hline $\begin{array}{l}\text { Dependent } \\
\text { variable }\end{array}$ & \multicolumn{14}{|c|}{ Annual cumulative length of inpatient-stay } \\
\hline $\begin{array}{l}\text { Regressor: } \\
\text { Time }\end{array}$ & -0.55 & 0.009 & 0.03 & 0.933 & -0.94 & 0.022 & -1.34 & 0.039 & 0.54 & 0.270 & -0.42 & 0.278 & -0.03 & 0.975 \\
\hline Constant & 45.7 & 0.000 & 27.4 & 0.000 & 32.2 & 0.000 & 63.6 & 0.000 & 51.5 & 0.000 & 26.5 & 0.000 & 65 & 0.000 \\
\hline $\begin{array}{l}\text { Dependent } \\
\text { variable }\end{array}$ & \multicolumn{14}{|c|}{ Proportion of readmitted individuals } \\
\hline $\begin{array}{l}\text { Regressor: } \\
\text { Time }\end{array}$ & -0.008 & 0.000 & 0.003 & 0.366 & -0.01 & 0.005 & 0.004 & 0.876 & -0.001 & 0.657 & -0.01 & 0.006 & -0.005 & 0.151 \\
\hline Constant & 0.44 & 0.000 & 0.21 & 0.000 & 0.52 & 0.000 & 0.41 & 0.000 & 0.34 & 0.000 & 0.28 & 0.000 & 0.56 & 0.000 \\
\hline $\begin{array}{l}\text { Dependent } \\
\text { variable }\end{array}$ & \multicolumn{14}{|c|}{ Proportion of high-frequently readmitted individuals ( $\geqslant 3$ readmissions) } \\
\hline $\begin{array}{l}\text { Regressor: } \\
\text { Time }\end{array}$ & -0.009 & 0.000 & -0.001 & 0.490 & -0.013 & 0.000 & -0.003 & 0.093 & -0.005 & 0.006 & -0.005 & 0.074 & -0.01 & 0.077 \\
\hline Constant & 0.17 & 0.000 & 0.02 & 0.034 & 0.24 & 0.000 & 0.13 & 0.000 & 0.10 & 0.000 & 0.07 & 0.000 & 0.29 & 0.000 \\
\hline
\end{tabular}

$b=$ regression coefficient; $p$-value $=$ statistical significance. Constant $=\mathrm{b}$; Italics $=$ level of significance $p<0.05$.

Bivariate regression analyses for annual number of outpatients treated and for six hospitalization variables with time as regressor showed a statistically significant change over the survey period for the majority of assessed variables. Annual number of outpatients treated increased significantly in the survey period for the whole sample $(b=67.3 ; p=0.038)$, and for patients suffering from organic mental disorders ( $b=46.1 ; p=0.000)$, mood disorders ( $b=58.4 ; p=0.037$ ), and personality disorders ( $b=12.6 ; p=0.001$ ), whereas the trend for those suffering from addictive disorders was a decreasing one ( $b=-32.8$; $p=0.001$ ). Number of annual admissions showed no significant tendencies for the whole sample, but there was a decreasing tendency for patients suffering from addictive disorders $(p=0.045)$ and schizophrenia $(p=0.000)$, contrary to Published by Sciedu Press 
those suffering from organic mental disorders $(p=0.000)$ and mood disorders $(p=0.045)$. Average number of readmissions decreased for the whole sample $(b=-0.06 ; p=0.001)$, especially for patients suffering from addictive $(p=0.009)$, mood ( $p=0.001)$ and adjustment and stress disorders $(p=0.021)$. Average length of inpatient-stay increased for the whole sample ( $b=0.31 ; p=0.000$ ), but decreased for patients suffering from schizophrenia $(p=0.000)$. On the other hand, annual cumulative length of inpatient-stay decreased over the surveyed period for the whole sample $(b=-0.55 ; p=0.009)$, especially for patients suffering from addictive disorders $(p=0.022)$ and schizophrenia $(p=0.039)$. Both proportion of readmissions $(b=-0.008 ; p=0.000)$ and proportion of frequently readmitted patients $(b=-0.009 ; p=0.000)$ decreased, especially for patients suffering from addictive disorders (see Table 4).

Table 5. Prais-Winsten regression models for time series (relevant inpatient variables) on the basis of robust regression coefficients according to principal diagnosis groups with number of outpatient treated as regressor

\begin{tabular}{|c|c|c|c|c|c|c|c|c|c|c|c|c|c|c|}
\hline \multirow{2}{*}{$\begin{array}{l}\text { Dependent variables } \\
\text { Diagnosis }\end{array}$} & \multicolumn{7}{|c|}{ Number of annual hospitalizations } & \multicolumn{7}{|c|}{ Average number of readmissions per readmitted individual } \\
\hline & $\begin{array}{l}\text { Sample } \\
\text { b (p) }\end{array}$ & $\begin{array}{l}\text { F0 } \\
\text { b (p) }\end{array}$ & $\begin{array}{l}\text { F1 } \\
\text { b (p) }\end{array}$ & $\begin{array}{l}\text { F2 } \\
\text { b (p) }\end{array}$ & $\begin{array}{l}\text { F3 } \\
\text { b (p) }\end{array}$ & $\begin{array}{l}\text { F4 } \\
\text { b (p) }\end{array}$ & $\begin{array}{l}\text { F6 } \\
\text { b (p) }\end{array}$ & $\begin{array}{l}\text { Sample } \\
\text { b (p) }\end{array}$ & $\begin{array}{l}\text { F0 } \\
\text { b (p) }\end{array}$ & $\begin{array}{l}\text { F1 } \\
\text { b (p) }\end{array}$ & $\begin{array}{l}\text { F2 } \\
\text { b (p) }\end{array}$ & $\begin{array}{l}\text { F3 } \\
\text { b (p) }\end{array}$ & $\begin{array}{l}\text { F4 } \\
\text { b (p) }\end{array}$ & $\begin{array}{l}\text { F6 } \\
\text { b (p) }\end{array}$ \\
\hline Outpatients treated & $\begin{array}{l}-0.09 \\
(0.041)\end{array}$ & $\begin{array}{l}0.58 \\
(0.000)\end{array}$ & $\begin{array}{l}-0.21 \\
(0.770)\end{array}$ & $\begin{array}{l}-0.23 \\
(0.126)\end{array}$ & $\begin{array}{l}0.11 \\
(0.526)\end{array}$ & $\begin{array}{l}0.06 \\
(0.836)\end{array}$ & $\begin{array}{l}0.75 \\
(0.004)\end{array}$ & $\begin{array}{l}-5 e-4 \\
(0.006)\end{array}$ & $\begin{array}{l}-4 \mathrm{e}-5 \\
(0.751)\end{array}$ & $\begin{array}{l}0.001 \\
(0.090)\end{array}$ & $\begin{array}{l}-0.001 \\
(0.125)\end{array}$ & $\begin{array}{l}-5 e-4 \\
(0.021)\end{array}$ & $\begin{array}{l}-0.002 \\
(0.029)\end{array}$ & $\begin{array}{l}-0.004 \\
(0.334)\end{array}$ \\
\hline Constant & $\begin{array}{l}3,830 \\
(0.000)\end{array}$ & $\begin{array}{l}-220 \\
(0.001)\end{array}$ & $\begin{array}{l}1,557 \\
(0.001)\end{array}$ & $\begin{array}{l}704 \\
(0.000)\end{array}$ & $\begin{array}{l}627 \\
(0.069)\end{array}$ & $\begin{array}{l}211 \\
(0.074)\end{array}$ & $\begin{array}{l}54.4 \\
(0.192)\end{array}$ & $\begin{array}{l}4.13 \\
(0.000)\end{array}$ & $\begin{array}{l}1.36 \\
(0.000)\end{array}$ & $\begin{array}{l}1.79 \\
(0.002)\end{array}$ & $\begin{array}{l}3.20 \\
(0.003)\end{array}$ & $\begin{array}{l}2.28 \\
(0.000)\end{array}$ & $\begin{array}{l}2.08 \\
(0.000)\end{array}$ & $\begin{array}{l}3.59 \\
(0.002)\end{array}$ \\
\hline$N / R^{2}$ & $9 /-$ & $9 / 0.89$ & $9 / 0.70$ & $9 / 0.88$ & $9 /-$ & $9 / 0.46$ & $9 / 0.86$ & $9 / 0.85$ & $9 / 0.95$ & $9 / 0.27$ & $9 / 0.34$ & $9 / 0.65$ & $9 / 0.32$ & 9/- \\
\hline$F /$ Prob $>F$ & $\begin{array}{r}6.25 / \\
0.041\end{array}$ & $\begin{array}{l}79 / \\
0.000\end{array}$ & $\begin{array}{l}0.09 / \\
0.770\end{array}$ & $\begin{array}{l}3 / \\
0.125\end{array}$ & $\begin{array}{l}0.45 / \\
0.526\end{array}$ & $\begin{array}{r}0.05 / \\
0.836\end{array}$ & $\begin{array}{l}16.9 / \\
0.004\end{array}$ & $\begin{array}{r}14.6 / \\
0.006\end{array}$ & $\begin{array}{c}0.11 / \\
0.750\end{array}$ & $\begin{array}{l}3.85 / \\
0.090\end{array}$ & $\begin{array}{l}3.03 / \\
0.125\end{array}$ & $\begin{array}{l}8.82 / \\
0.021\end{array}$ & $\begin{array}{l}7.5 / \\
0.029\end{array}$ & $\begin{array}{r}1.07 / \\
0.334\end{array}$ \\
\hline \multirow[t]{2}{*}{$R h o / D-W_{t}$} & $\begin{array}{c}-0.01 / \\
1.84\end{array}$ & $\begin{array}{l}-0.48 / \\
2.02\end{array}$ & $\begin{array}{c}0.52 / \\
1.71\end{array}$ & $\begin{array}{c}0.83 / \\
1.48\end{array}$ & $\begin{array}{l}0.72 / \\
1.00\end{array}$ & $\begin{array}{c}0.29 / \\
1.23\end{array}$ & $\begin{array}{l}-0.47 / \\
1.61\end{array}$ & $\begin{array}{l}0.57 / \\
1.63\end{array}$ & $\begin{array}{c}-0.40 / \\
1.24\end{array}$ & $\begin{array}{c}-0,38 / \\
1.83\end{array}$ & $\begin{array}{c}-0.03 / \\
1.77\end{array}$ & $\begin{array}{l}0.06 / \\
1.63\end{array}$ & $\begin{array}{l}-0.05 / \\
1.83\end{array}$ & $\begin{array}{c}-0.24 / \\
1.39\end{array}$ \\
\hline & \multicolumn{7}{|c|}{ Average length of inpatient-stay } & \multicolumn{7}{|c|}{ Average annual cumulative length of inpatient-stay } \\
\hline Outpatients treated & $\begin{array}{l}9 \mathrm{e}-4 \\
(0.113)\end{array}$ & $\begin{array}{l}0.002 \\
(0.627)\end{array}$ & $\begin{array}{l}9 \mathrm{e}-4 \\
(0.537)\end{array}$ & $\begin{array}{l}-5 e-3 \\
(0.550)\end{array}$ & $\begin{array}{l}0.013 \\
(0.065)\end{array}$ & $\begin{array}{l}-0.002 \\
(0.745)\end{array}$ & $\begin{array}{l}0.035 \\
(0.109)\end{array}$ & $\begin{array}{l}-6 e-3 \\
(0.018)\end{array}$ & $\begin{array}{l}11.9 \\
(0.000)\end{array}$ & $\begin{array}{l}1.04 \\
(0.893)\end{array}$ & $\begin{array}{l}-4.07 \\
(0.331)\end{array}$ & $\begin{array}{l}3.81 \\
(0.673)\end{array}$ & $\begin{array}{l}1.08 \\
(0.699)\end{array}$ & $\begin{array}{l}21.22 \\
(0.042)\end{array}$ \\
\hline Constant & $\begin{array}{l}16.8 \\
(0.000)\end{array}$ & $\begin{array}{l}19.3 \\
(0.000)\end{array}$ & $\begin{array}{l}10.8 \\
(0.000)\end{array}$ & $\begin{array}{l}33.6 \\
(0.003)\end{array}$ & $\begin{array}{l}17.4 \\
(0.023)\end{array}$ & $\begin{array}{l}17.3 \\
(0.000)\end{array}$ & $\begin{array}{l}15.9 \\
(0.005)\end{array}$ & $\begin{array}{l}67 \\
(0.000)\end{array}$ & $\begin{array}{l}-4,557 \\
(0.000)\end{array}$ & $\begin{array}{l}19,972 \\
(0.002)\end{array}$ & $\begin{array}{l}18,774 \\
(0.006)\end{array}$ & $\begin{array}{l}19,510 \\
(0.164)\end{array}$ & $\begin{array}{l}3,439 \\
(0.013)\end{array}$ & $\begin{array}{l}410 \\
(0.829)\end{array}$ \\
\hline$N / R^{2}$ & $9 / 0.95$ & $9 / 0.83$ & $9 / 0.80$ & $9 / 0.72$ & $9 / 0.34$ & $9 /-$ & $9 / 0.47$ & $9 / 0.16$ & $9 / 0.97$ & $9 / 0.69$ & $9 / 0.85$ & $9 / 0.69$ & $9 / 0.699$ & $9 / 0.38$ \\
\hline$F /$ Prob $>F$ & $\begin{array}{l}3.3 / \\
0.133\end{array}$ & $\begin{array}{l}0.26 / \\
0.627\end{array}$ & $\begin{array}{l}0.42 / \\
0.537\end{array}$ & $\begin{array}{l}0.39 / \\
0.549\end{array}$ & $\begin{array}{l}4.76 / \\
0.065\end{array}$ & $\begin{array}{c}0.11 / \\
0.745\end{array}$ & $\begin{array}{l}3.38 / \\
0.108\end{array}$ & $\begin{array}{l}9.4 / \\
0.018\end{array}$ & $\begin{array}{l}219 / \\
0.000\end{array}$ & $\begin{array}{l}0.02 / \\
0.892\end{array}$ & $\begin{array}{l}1.09 / \\
0.673\end{array}$ & $\begin{array}{l}0.19 / \\
0.673\end{array}$ & $\begin{array}{l}0.16 / \\
0.699\end{array}$ & $\begin{array}{c}6.13 / \\
0.042\end{array}$ \\
\hline \multirow[t]{2}{*}{$R h o / D-W_{t}$} & $\begin{array}{l}0.67 / \\
1.29\end{array}$ & $\begin{array}{l}-0.20 / \\
2.00\end{array}$ & $\begin{array}{l}-0.21 / \\
1.52\end{array}$ & $\begin{array}{c}0.19 / \\
1.73\end{array}$ & $\begin{array}{l}0.005 / \\
1.88\end{array}$ & $\begin{array}{l}0.005 / \\
1.37\end{array}$ & $\begin{array}{l}-0.07 / \\
1.71\end{array}$ & $\begin{array}{l}-0.12 / \\
1.67\end{array}$ & $\begin{array}{l}-0.76 / \\
2.44\end{array}$ & $\begin{array}{l}0.52 / \\
1.71\end{array}$ & $\begin{array}{l}0.73 / \\
1.43\end{array}$ & $\begin{array}{c}0.73 / \\
1.43\end{array}$ & $\begin{array}{c}0.43 / \\
1.23\end{array}$ & $\begin{array}{l}0.18 / \\
1.45\end{array}$ \\
\hline & \multicolumn{7}{|c|}{ Proportion of readmitted individuals } & \multicolumn{7}{|c|}{ Proportion of frequently readmitted patients ( $\geqslant 3$ readmissions) } \\
\hline Outpatients treated & $\begin{array}{l}-5 e-5 \\
(0.020)\end{array}$ & $\begin{array}{l}4 \mathrm{e}-5 \\
(0.548)\end{array}$ & $\begin{array}{l}1 \mathrm{e}-4 \\
(0.395)\end{array}$ & $\begin{array}{l}1 \mathrm{e}-4 \\
(0.395)\end{array}$ & $\begin{array}{l}-7 e-5 \\
(0.040)\end{array}$ & $\begin{array}{l}-5 e-4 \\
(0.007)\end{array}$ & $\begin{array}{l}2 \mathrm{e}-4 \\
(0.352)\end{array}$ & $\begin{array}{l}-4 \mathrm{e}-5 \\
(0.065)\end{array}$ & $\begin{array}{l}-2 \mathrm{e}-5 \\
(0.389)\end{array}$ & $\begin{array}{l}3 e-4 \\
(0.006)\end{array}$ & $\begin{array}{l}-9 \mathrm{e}-5 \\
(0.243)\end{array}$ & $\begin{array}{l}-9 e-5 \\
(0.006)\end{array}$ & $\begin{array}{l}2 \mathrm{e}-4 \\
(0.089)\end{array}$ & $\begin{array}{l}5 e-4 \\
(0.210)\end{array}$ \\
\hline Constant & $\begin{array}{l}0.62 \\
(0.000)\end{array}$ & $\begin{array}{l}0.20 \\
(0.001)\end{array}$ & $\begin{array}{l}0.54 \\
(0.000)\end{array}$ & $\begin{array}{l}0.54 \\
(0.000)\end{array}$ & $\begin{array}{l}0.41 \\
(0.000)\end{array}$ & $\begin{array}{l}0.38 \\
(0.000)\end{array}$ & $\begin{array}{l}0.58 \\
(0.000)\end{array}$ & $\begin{array}{l}0.29 \\
(0.005)\end{array}$ & $\begin{array}{l}0.03 \\
(0.105)\end{array}$ & $\begin{array}{l}0.05 \\
(0.123)\end{array}$ & $\begin{array}{l}0.19 \\
(0.015)\end{array}$ & $\begin{array}{l}0.17 \\
(0.000)\end{array}$ & $\begin{array}{l}0.12 \\
(0.010)\end{array}$ & $\begin{array}{l}0.33 \\
(0.002)\end{array}$ \\
\hline$N / R^{2}$ & $9 / 0.96$ & $9 / 0.89$ & $9 / 0.89$ & $9 / 0.89$ & $9 / 0.82$ & $9 / 0.09$ & 9/- & $9 / 0.80$ & $9 / 0.03$ & $9 / 0.03$ & $9 / 0.13$ & $9 / 0.65$ & $9 / 0.23$ & $9 / 0.13$ \\
\hline$F /$ Prob $>F$ & $\begin{array}{l}9.9 / \\
0.020\end{array}$ & $\begin{array}{l}0.40 / \\
0.548\end{array}$ & $\begin{array}{l}0.82 / \\
0.395\end{array}$ & $\begin{array}{l}1.14 / \\
0.322\end{array}$ & $\begin{array}{l}6.34 / \\
0.040\end{array}$ & $\begin{array}{r}13.9 / \\
0.007\end{array}$ & $\begin{array}{l}1.0 / \\
0.352\end{array}$ & $\begin{array}{l}5.0 / \\
0.060\end{array}$ & $\begin{array}{r}0.84 / \\
0.389\end{array}$ & $\begin{array}{l}15.6 / \\
0.005\end{array}$ & $\begin{array}{r}1.62 / \\
0.243\end{array}$ & $\begin{array}{l}15.3 / \\
0.006\end{array}$ & $\begin{array}{l}3.9 / \\
0.089\end{array}$ & $\begin{array}{c}1.90 / \\
0.210\end{array}$ \\
\hline$R h o / D-W_{t}$ & $\begin{array}{l}0.63 / \\
1.44\end{array}$ & $\begin{array}{l}-0.55 / \\
1.46\end{array}$ & $\begin{array}{l}0.74 / \\
1.67\end{array}$ & $\begin{array}{l}-0,356 / \\
2.12\end{array}$ & $\begin{array}{c}0.31 / \\
1.36\end{array}$ & $\begin{array}{l}-0.24 / \\
1.51\end{array}$ & $\begin{array}{l}-0.01 / \\
1.32\end{array}$ & $\begin{array}{c}0.66 / \\
1.36\end{array}$ & $\begin{array}{c}0.09 / \\
1.79\end{array}$ & $\begin{array}{l}-0.20 / \\
1.85\end{array}$ & $\begin{array}{l}-0.08 / \\
1.92\end{array}$ & $\begin{array}{l}-0.29 / \\
2.24\end{array}$ & $\begin{array}{l}-0.17 / \\
1,89\end{array}$ & $\begin{array}{l}-0.04 / \\
1.46\end{array}$ \\
\hline
\end{tabular}

$b=$ regression coefficient; $p=$ statistical significance. Constant $=\mathrm{b}_{0} ; \mathrm{N}=$ Time series. $\mathrm{R}^{2}=$ fit of regression analysis as degree of explanation effect. $\mathrm{F}=$ Value in F-distribution. Rho= Iteration coefficient. $\mathrm{D}$-Wt $=$ transformed Durbin-Watson coefficient d (test on residual correlation time series). Italics= statistically significant ( $\mathrm{p}<0.05$ level)

Bivariate regression analyses for time series demonstrated a negative association between number of outpatients treated and number of annual admissions ( $p=0.041)$, average number of readmissions ( $p=0.006)$, annual cumulative length of inpatient-stay $(p=0.018)$, and proportion of readmitted individuals $(p=0.020)$ (see Table 5 ). Analyses according to diagnostic groups make more distinctions. The number of annual outpatients treated and number of annual admissions were positively associated for patients suffering from organic mental disorders $(p=0.000)$ and personality disorders $(p=0.004)$. Number of outpatients treated was negatively associated with average number of readmissions for individuals suffering from mood disorders $(p=0.021)$ and those suffering from adjustment and stress disorders $(p=0.029)$. There was no significant association for any diagnosis with average length on inpatient-stay but a positive association with annual cumulative length of inpatient-stay for individuals suffering from organic mental disorders $(p=0.000)$ and personality disorders ( $p=0.042$ ). Number of outpatients treated was negatively associated with proportion of readmitted individuals for patients suffering from mood $(p=0.040)$ and adjustment and stress disorders $(p=0.007)$, whereas proportion of frequently readmitted patients was positively associated for patients suffering from addictive disorders $(p=0.006)$ and negatively associated for those suffering from mood disorders ( $p=0.006$ ) (see Table 5). 


\section{Discussion}

This German investigation demonstrates that even after conclusion of a deinstitutionalization process, number of admissions and admitted individuals may remain stable, whereas readmission figures, and annual cumulative length of inpatient-stay per individual may decrease depending on the activity of a community-based outpatient clinic. These effects were more accurately demonstrated for patients suffering from addictive disorders. According to the results of other investigations, number of admissions, readmission figures and cumulative length of inpatient-stay do not necessarily increase when the number of beds decreases and admission pressure nationwide increases (overview in ${ }^{[11]}$ ). Häfner \& Heiden ${ }^{[25]}$ demonstrated for a German cohort of schizophrenic patients that outpatient care had a significant influence on readmission, but no effect on the length of inpatient treatment.

We compared the main results of our investigation with figures for the state (Bavaria) and federal (Germany) levels in order to capture different developments and tendencies. Within the survey period, number of psychiatric admissions increased in Germany ${ }^{[4]}$ from 986,573 to 1,163,613, or about 18\%, and in Bavaria from 143,276 to 180,121, or about 26\%, whereas in the surveyed catchment area number of admissions remained stable. Admission rates also increased at federal and state level, whereas they even decreased in the catchment area (see Table 3). Average length of inpatient-stay decreased at the beginning of the survey period faster in the catchment area, but increased until 2010 by about two days, putting it on the same level as Germany and Bavaria (see Table 3). There are also striking differences in development of diagnostic distribution in hospitalized patients. Whereas in Germany the proportion of principal psychiatric diagnosis remained stable, organic mental disorders increased and addictive disorders decreased substantially in the surveyed area, mood disorders increased and schizophrenia decreased everywhere; in the surveyed area, there were lower numbers of organic mental disorders as well as adjustment and stress disorders, but higher numbers of addictive and personality disorders than at federal level (see Table 2). Average length of inpatient-stay remained lower in the surveyed area than at federal level for patients suffering from schizophrenia, adjustment and stress disorders as well as personality disorders, contrary to those suffering from organic mental disorders and addictive disorders (see Table 3). Cumulative individual data are not available in German registers. It is remarkable that even involuntary admissions under the guardianship law remain clear under the federal and state levels despite a slight increase in the last three years of the survey period, perhaps because efforts to provide alternatives to coercion are more efficient in rural areas on the basis of personalized networking ${ }^{[26]}$. The fact that inpatient coercive measures also decreased (see Table 1 ) may be explained by mediating effects. The hypothesis that intensive community-based outpatient care by an outpatient clinic may control inpatient variables such as number of admissions, readmissions, cumulative length of inpatient-stay and even coercion may be reinforced by the fact that annual prevalence of treatment periods in the surveyed catchment area per 1,000 inhabitants is three times higher than the average figures in the state (Bavaria) in which assessed catchment area is placed (see Table 3).

Bivariate regression analyses for time series referring to the whole surveyed sample demonstrate that annual number of outpatients treated increased, number of admissions remained stable, and annual cumulative length of inpatient-stay as well as readmission figures decreased significantly, contrary to average length of inpatient-stay per hospitalization. As regards principal diagnostic groups, patients suffering from organic mental disorders showed increasing outpatient as well as hospitalization figures, probably because of epidemiological factors related to an ageing population. Individuals suffering from addictive disorders showed decreasing number of admissions and readmissions, perhaps because care continuity by outpatient clinic after discharge improved over time. The reduction of hospitalizations, average length of inpatient-stay and cumulative length of inpatient-stay in patients suffering from schizophrenia demonstrate that after conclusion of the deinstitutionalization process, avoidance of the revolving-door phenomenon is possible when community-based treatment is ensured. The number of patients suffering from mood disorders increased in both inpatient and outpatient settings, whereas readmission figures decreased as a positive effect of continuity of care after discharge. Patients suffering from adjustment and stress disorders only show decreasing readmission figures, whereas all assessed hospitalization variables remain stable for patients suffering from personality disorders. We assume there is a diagnostic 
shift from personality as well as adjustment and stress disorders to mood disorders because of a better social acceptance of 'mood disorder' as a psychiatric diagnosis. Valdes-Stauber \& Kilian ${ }^{[27]}$ recently demonstrated that patients suffering from personality disorders needed three times as much annual contact time as those suffering from schizophrenia and six times as much as those suffering from affective or adjustment and stress disorders.

Bivariate regression analyses between number of annual outpatients treated and hospitalization variables show that increasing community-based care may reduce the number of admissions, readmissions and cumulative length of inpatient-stay but not average length of inpatient-stay. It seems, however, that an increase in outpatient activity leads to more admissions and to a higher cumulative length of inpatient-stay in patients suffering from addictive and personality disorders, perhaps because a stronger awareness of acute situations leads to more intensive treatment decisions like hospitalization.

The findings of this study suggest that the role of the outpatient clinics in control hospitalization is decisive. Zhang, Harvey \& Andrew ${ }^{[28]}$ found that quality of inpatient care does not affect the risk of readmission, but that more active and assertive treatment in the community post-discharge decreases the risk of readmission. A Danish survey ${ }^{[29]}$ demonstrated, however, that a series of negative indicators appeared with the deinstitutionalization process such as increase of suicides, in the number of the criminal mentally disordered, in coercive activities in the wards and in bed occupancy, all related to a dramatic reduction of psychiatric beds. The question of adequate length of inpatient-stay in psychiatric care has been hotly discussed since the late 1990s. Long stays and readmissions seem to be less necessary when well-functioning community-based teams ensure continuity of care ${ }^{[13]}$, but some investigations showed that over-brief hospitalizations risk patients being readmitted ${ }^{[30,31]}$. Lieberman et al. ${ }^{[32]}$ point out that improvement during very brief hospitalizations is comparable to that in longer stays in many ways; however, depressed patients discharged more quickly show significantly higher residual levels of depressive symptoms and lower levels of global functioning, which may place them at greater risk of adverse outcomes in the immediate post-hospital period. This risk was also found for schizophrenia ${ }^{\text {[3] }}$ and for psychogeriatric patients ${ }^{[34]}$.

The results of this investigation suggest that community-based outpatient activity can reduce readmissions and cumulative length of inpatient-stay as well as control the number of hospitalizations, even after concluding deinstitutionalization and after closing long-stay wards. According to the literature, there is strong evidence that social factors contribute to the duration of the hospital stay and to frequent rehospitalization ${ }^{[35-37]}$ as well as the lack of continuity of care after discharge ${ }^{[38]}$. In turn, non-adherence is related to an involuntary legal status at discharge or rejection of medical advice, not having an established outpatient clinician, social problems within the primary support group and the number of days between hospital discharge and follow-up appointment ${ }^{[38]}$. These results indicate the scope of the tasks which need to be completed by outpatient teams in order to avoid the risk of early rehospitalization: continuity of care by avoiding a gap between discharge and first appointment, relieving social burdens, offering assertive care and supporting and advocating relationships for patients with a lack of insight or denial of their illness. Otherwise, the benefits of intensive care management could be marginal in settings that have already achieved low rates of bed use; according to Burns et al. ${ }^{[39]}$, it might not be necessary to apply the full model of assertive community treatment to achieve reductions in hospitalization figures.

According to a previous multivariate analysis, ambulatory assertive treatment is associated with decreasing inpatient restraint measures and even with proportion of involuntary admissions, perhaps because there are intrinsic boundaries in the application of force by medical institutions ${ }^{[40]}$. It seems that there is a tendency to limit the application of further forceful measures after an involuntary admission. The continuity of care after admission through cooperation with psychiatric wards may reduce distress and fear and consequently reduce the application of force in psychiatry ${ }^{[5]}$. Monitored data collection related to coercive measures may improve awareness concerning the application of forcible measures; this process could itself reduce such measures in psychiatry. Otherwise, community-based outpatient services are based on a network of balanced statutory and non-statutory stakeholders and authorities. For this reason cooperation 
with legal guardians, institutions, authorities and especially judges is a constructive way to decrease admissions and search for alternatives to involuntary admissions. Such cooperation seems to be more difficult within the realm of commitment law, owing to autonomous police work and the emergence of acute situations primarily independent of medical professionals, because of their shift work schedules, different settings and different legal frames and because communication with patients is so difficult ${ }^{[41]}$. Therefore the avoidance of involuntary admissions by police is a more difficult matter, especially when there is a lack of professionals on duty to assess police and on-site carers ${ }^{[26]}$. Correlations between coercive measures and hospital characteristics were widely investigated in Germany by Steinert et al. ${ }^{[42]}$ in longitudinal and comparative surveys.

The results of our investigation confirm findings of other investigations which demonstrate that deinstitutionalization does not necessarily lead to increases in number of hospitalizations, cumulative length of inpatient-stay, readmission figures or number of coercive measures ${ }^{[43,44]}$, contrary to findings that deinstitutionalization leads to more readmissions (revolving-door phenomenon), increasing cumulative length of inpatient-stay and even more involuntary admissions ${ }^{[45]}$. According to Szmukler \& Holloway ${ }^{[11]}$, an increase in involuntary hospitalizations is not an inevitable consequence of reducing psychiatric beds. Community-based ambulatory care by multi-disciplinary teams with a focus on quality of relationship and support for carers seems capable of reducing rehospitalizations and controlling cumulative length of inpatient-stay and frequency of coercion measures.

\section{References}

[1] Rössler, W., \& Salize, H.J. Longitudinal statistics of mental health care in Germany. Social Psychiatry and Psychiatric Epidemiology. 1994; 29: 112-118. PMid:8085179

[2] Hohagen, F. The Declaration of Helsinki- A challenge for German psychiatry (Die Deklaration von Helsinki- eine Herausforderung für die deutsche Psychiatrie). Der Nervenarzt. 2006; 77: 1133-1142.

[3] Schneider, F., Falkai, P., \& Maier, W. (2011). Report about German psychiatry in the year 2020 by the German Association of Psychiatry (DGPPN). Berlin, Heidelberg: Springer. [German]

[4] German Federal Health Report. (2011) [Internet]. (Gesundheitsberichterstattung des Bundes). Available from: www.gbe-bund.de. [German]

[5] Valdes-Stauber, J., Kilian, R., \& Deinert, H. German guardianships, involuntary admissions and physical restraint measures in psychiatry: Implications for community-based psychiatry. In I. Needham, H. Nijman, T. Palmstierna, R. Almvik, \& N. Oud (Eds.) Proceedings of the 7th European Congress on Violence in Clinical Psychiatry (pp. 99-102). Amsterdam: Kavanah. 2011.

[6] Federal Joint Commission of Stakeholders of Psychiatric Hospitals in Germany (Bundesarbeitsgemeinschaft der Träger Psychiatrischer Krankenhäuser) (2009). Survey on psychiatric out-patient services in Germany. DKG (German Hospital Society). [German]

[7] Valdes-Stauber, J. Reflection on the establishment of outpatient services in Germany considering real German psychiatric care (Beitrag zu einer Standortbestimmung der psychiatrischen Institutsambulanzen in der deutschen psychiatrischen Versorgung). Sozialpsychiatrische Informationen. 2010; 40: 20-25. [German]

[8] Becker, Th. Out-patient psychiatric services. In G. Thornicroft, \& G. Szmukler (Eds) Textbook of community psychiatry (pp. 277-282). Oxford, New York: Oxford University Press. 2001

[9] Goldberg, D., Jackson, G., Gater, R., Campbell, M., \& Jennett, N. The treatment of common mental disorders by a community team based in primary care: a cost-effectiveness study. Psychological Medicine. 1996; 26: 487-492. PMid:8733207 http://dx.doi.org/10.1017/S003329170003556X

[10] Lehmann, A.F., Dixon, L.B., Kernan, E., DeForge, B.R., \& Postrado, L.T. A randomized trial of assertive community treatment for homeless persons with severe mental illness. Archives of General Psychiatry. 1997; 54: 1038-1043. http://dx.doi.org/10.1001/archpsyc.1997.01830230076011

[11] Szmukler, G., \& Holloway F. In-patient treatment. In G. Thornicroft, \& G. Szmukler (Eds) Textbook of community psychiatry (pp. 321-337). Oxford, New York: Oxford University Press. 2001.

[12] Burns, T., Knapp, M., Catty, J., Healey, A., Henderson, J., Watt, H., \& Wright, C. Home treatment for mental health problems: a systematic review. Health Technologies Assessment Journal. 2000; 5: 1-139.

[13] Marshall, M., \& Lockwood, A. (2000). Assertive community treatment for people with severe mental disorders. Cochrane Database Systematic Reviews, 2, CD001089. PMid:10796415 
[14] Marshall, M., Crowther, R., Almaraz-Serrano, A.M., \& Tyrer, P. (2001). Day hospital versus out-patient care for psychiatric disorders. Cochrane Database Systematic Review, 3, CD003240. PMid:11687059 http://dx.doi.org/10.1002/14651858.CD003240

[15] Robin, M., Bronchard, M., \& Kannas, S. Ambulatory care provision versus first admission to psychiatric hospital: 5 years follow up. Social Psychiatry and Psychiatric Epidemiology. 2008; 43: 498-506. PMid:18320129 http://dx.doi.org/10.1007/s00127-008-0326-0

[16] Valevski A., Olfson M., Weizman A., \& Shiloh R. Risk of readmission in compulsorily and voluntarily admitted patients. Social Psychiatry and Psychiatric Epidemiology. 2007; 42: 916-922. PMid:17712501 http://dx.doi.org/10.1007/s00127-007-0243-7

[17] Burgess, P., Bindmann, J., Leese, M., Henderson, C., \& Szmukler, G. Do community treatment orders for mental illness reduce readmission to hospital? An epidemiological study. Social Psychiatry and Psychiatric Epidemiology. 2006; 41: 574-579. PMid:16685479 http://dx.doi.org/10.1007/s00127-006-0063-1

[18] Segal, S.P., \& Burgess, P.M. Use of community treatment orders to prevent psychiatric hospitalization. Australian and New Zealand Journal of Psychiatry. 2008; 42: 732-739. PMid:18622781 http://dx.doi.org/10.1080/00048670802206312

[19] Tyrer, P., Evans, K., Gandhi, N., Lamont, A., Harrison-Read, P., \& Johnson, T. Randomised controlled trial of two models of care for discharged psychiatric patients. British Medical Journal. 1998; 316: 106-109. PMid:9462315 http://dx.doi.org/10.1136/bmj.316.7125.106

[20] Saarento, O., Kastrup, M., Lönnerberg, O., Göstas, G., Muus, S., Sandlund, M., Öiesvold, T., \& Hansson, L. The Nordic Comparative Study on Sectorized Psychiatry: patients who use only psychiatric in-patient care in comprehensive community-based services-a 1-year follow-up study. Acta Psychiatrica Scandinavica. 1998; 98: 98-104. PMid:9718234 http://dx.doi.org/10.1111/j.1600-0447.1998.tb10049.x

[21] Prais, S. J., \& Winsten, C.B. (1954) Trend estimators and serial correlation. Working paper 383, Cowles Commission.

[22] Judge, G. G., Griffiths, W. E., Hill, R. C., Lütkepohl, H., \& Lee T.-C. The theory and practice of econometrics. 2nd ed. New York: Wiley. 1985.

[23] Durbin, J., \& Watson, G. S. Testing for serial correlation in least squares regression. I. Biometrika. 1950; 37: 409-428. PMid:14801065 http://dx.doi.org/10.2307/2332391

[24] StataCorp. Stata Statistical Software. Release 12. College Station, TX: StataCorp LP. 2011.

[25] Häfner, H., \& an der Heiden, W. The evaluation of mental health care systems. British Journal of Psychiatry. 1989 ; $155:$ 12-17. PMid:2513999 http://dx.doi.org/10.1192/bjp.155.1.12

[26] Valdes-Stauber, J, Wiederholt, F., \& Kilian, R. Are there different tendencies in the ordering of care and confinement between urban and rural areas? A Bavarian comparison between Munich and Alpine Region. Psychiatrische Praxis. 2012; 39: $267-274$. PMid:22926791

[27] Valdes-Stauber, J., \& Kilian, R. Statutory duties of German psychiatric outpatient clinics and their real care conditions. The Case of a Bavarian rural outpatient clinic on example. Psychiatrische Praxis. 2013; 40: 146-153. PMid:23345189

[28] Zhang, J., Harvey, C., \& Andrew, C. Factors associated with length of stay and the risk of readmission in an acute psychiatric inpatient facility: a retrospective study. Australian and New Zealand Journal of Psychiatry. 2011; 45: 578-585. PMid:21718126 http://dx.doi.org/10.3109/00048674.2011.585452

[29] Munk-Jørgensen, P. Has deinstitutionalisation gone too far? European Archives of Psychiatry and Clinical Neurosciences. 1999; 249: 136-143. PMid:10433127 http://dx.doi.org/10.1007/s004060050078

[30] Spießl, H., Binder, H., Cording, C., Klein, H.E., \& Hajak, G. Psychiatric inpatient care under economic pressure. Deutsches Ärzteblatt. 2006; 103: A2549-52. [German]

[31] Niehaus, D.J., Koen, L., Galal, U., Dhansay, K., Oosthuizen, P.P., Emsley, R.A., \& Jordaan, E. Crisis discharges and readmission risk in acute psychiatric male inpatients. BioMedCentral Psychiatry. 2008; 8: 44.

[32] Lieberman P.B., Wiitala S.A., Elliott B., McCormick S., \& Goyette S.B. Decreasing length of stay: Are there effects on outcomes of psychiatric hospitallization? American Journal of Psychiatry. 1998; 155: 905-909. PMid:9659855

[33] Appleby, L., Luchins, D.J., Desai, P.N., Gibbons, R.D., Jamicak, R.G., \& Marks, R. Length of stay and recidivism among patients with schizophrenia. Psychiatric Services. 1996; 47: 985-990. PMid:8875666

[34] Heeren, O., Dixon, L., Gavirneni, S., \& Regenold, W.T. The association between decreasing length of stay and readmission rate on a psychogeriatric unit. Psychiatric Services. 2002; 53: 76-79. PMid:11773653 http://dx.doi.org/10.1176/appi.ps.53.1.76

[35] Kent, S., \& Yellowlees, P. Psychiatric and social reasons for frequent rehospitalization. Hospital and Community Psychiatry. 1994; 45: 347-350. PMid:8020919

[36] Blais, M.A., Matthews, J., Lipkis-Orlando, R., Lechner, E., Jacobo, M., Lincoln, R., Gulliver, C.H., Herman, J.B., \& Goodmann, A.F. Predicting length of stay on an acute care medical psychiatric inpatient service. Administration and Policy in Mental Health and Mental Health Research. 2003; 31: 15-29. http://dx.doi.org/10.1023/A:1026044106172 
[37] Abas, M.A., Vanderpyl, J., Robinson, E., Le Prou, T., \& Crampton, P. Socio-economic deprivation and duration of hospital stay in severe mental disorder. British Journal of Psychiatry. 2006; 188: 581-582. PMid:16738350 http://dx.doi.org/10.1192/bjp.bp.104.007476

[38] Compton, M.T., Rudisch, B.E., Craw, J., Thompson, T., \& Owens, D.A. Predictors of missed first appointments at community mental health centres after psychiatric hospitalization. Psychiatric Services. 2006; 57: 531-537. PMid:16603749 http://dx.doi.org/10.1176/appi.ps.57.4.531

[39] Burns, T., Catty, J., Dash, M., Roberts, Ch., Lockwood, A., \& Marshall, M. Use of intensive care management to reduce time in hospital in people with severe mental illness: systematic review and meta-regression. British Journal of Medicine. 2007; $335: 336$. PMid:17631513 http://dx.doi.org/10.1136/bmj.39251.599259.55

[40] Valdes-Stauber, J. Development of coercive measures in a German psychiatric hospital over a period of nine years. In I. Needham, K. McKenna, M. Kingma, \& N. Oud (Eds) Second International Conference on Violence in the Health Sector. From awareness to sustainable action (pp. 204-207). Amsterdam: Kavanah. 2010.

[41] Valdes-Stauber, J., Deinert, H., \& Kilian, R. German practice of involuntary commitment at both federal and state level after introduction of the guardianship law (1999-2009). Fortschritte der Neurologie und Psychiatrie. 2012; 80: 267-275. PMid:22116739 http://dx.doi.org/10.1055/s-0031-1281739

[42] Steinert, T., Martin, V., Baur, M., Bohnet, U., Goebel, R., Hermelink, G., Kromstorfer R., Kuster, W., Martínez-Funk, B., Roser, M., Schwink A., \& Voigtländer, W. Diagnosis-related frequency of compulsory measures in ten German psychiatric hospitals and correlates with hospital characteristics. Social Psychiatry and Psychiatric Epidemiology. 2007; 42: 140-145. PMid:17180296 http://dx.doi.org/10.1007/s00127-006-0137-0

[43] Wall, S., Hotopf, M., Wessely, S., \& Churchill, R. Trends in the use of the Mental Health Act: England, 1984-96. British Medical Journal. 1999; 318: 1520-1521. PMid:10356005 http://dx.doi.org/10.1136/bmj.318.7197.1520

[44] Fioritti, A., Lo, R.L., \& Melega, V. Reform said or done? The case of Emilia-Romagna within the Italian psychiatric context. American Journal of Psychiatry. 1997; 154: 94-98. PMid:8988965

[45] Tansella, M., Amaddeo, F., Burti, I., Garzotto, N., \& Ruggerim, M. Community-based mental health care in Verona, Italy. In D. Goldberg, \& G. Thornicroft (Eds.) Mental health in our future cities (pp. 239-262). Maudsley Monograph 42. Hove: Psychology Press. 1998. 